\title{
Dynamic Linear Precoding for the Exploitation of Known Interference in MIMO Broadcast Systems
}

\author{
Christos Masouros, Student Member, IEEE, and Emad Alsusa, Senior Member, IEEE
}

\begin{abstract}
This paper introduces a novel channel inversion (CI) precoding scheme for the downlink of phase shift keying (PSK)-based multiple input multiple output (MIMO) systems. In contrast to common practice where knowledge of the interference is used to eliminate it, the main idea proposed here is to use this knowledge to glean benefit from the interference. It will be shown that the system performance can be enhanced by exploiting some of the existent inter-channel interference (ICI). This is achieved by applying partial channel inversion such that the constructive part of ICI is preserved and exploited while the destructive part is eliminated by means of CI precoding. By doing so, the effective signal to interference-plus-noise ratio (SINR) delivered to the mobile unit (MU) receivers is enhanced without the need to invest additional transmitted signal power at the MIMO base station (BS). It is shown that the trade-off to this benefit is a minor increase in the complexity of the BS processing. The presented theoretical analysis and simulations demonstrate that due to the SINR enhancement, significant performance and throughput gains are offered by the proposed MIMO precoding technique compared to its conventional counterparts.
\end{abstract}

Index Terms-Adaptive signal processing, MIMO systems, interference multiuser channels, channel inversion, precoding.

\section{INTRODUCTION}

T HE increasing demand for high data rate multimedia transmission has recently stimulated a tremendous amount of research on multi-antenna communications [1] towards capacity enhancement of the communication links. Despite the increased capacity these schemes offer, due to the space diversity they add to the systems' optimization, it is well known that the spatial correlation of the MIMO sub-channels introduces additional interference which corrupts the data symbols and in effect degrades the achievable performance of such systems. MIMO detection techniques [2-5] have been traditionally applied to counteract this impediment. Despite their superior performance in high diversity multi-antenna systems, these techniques tend to involve quite complex detection processing which renders them impractical for use in the MUs in downlink communications. In order to shift the signal enhancement processing to the BS during downlink transmission, various precoding techniques have been developed with the view to maintain simple and cost-effective MUs. Channel inversion (CI), proposed in [6], offers the least complexity of the precoding techniques available. However, the disadvantages of the CI technique include a poor symbol

Manuscript received January 15, 2008; revised May 27, 2008 and July 31, 2008; accepted September 12, 2008. The associate editor coordinating the review of this paper and approving it for publication was R. Mallik.

The authors are with the School of Electrical \& Electronic Engineering, The University of Manchester, PO Box 88, Manchester, M16 1QD, UK (e-mail: chris.masouros@postgrad.manchester.ac.uk, e.alsusa@manchester.ac.uk).

Digital Object Identifier 10.1109/TWC.2009.080053 error rate (SER) performance and the fact that the transmission rate and throughput delivered are limited and do not improve with the increase in the number of antennas, as demonstrated in [7]. The solution proposed in [7], namely regularised channel inversion (RCI) provides some performance and capacity gains with respect to the conventional CI. Nevertheless, the transmission rates offered by this scheme are far from reaching the theoretical channel capacity. The need for further increase in transmission rate has led to the proposal of dirty paper coding (DPC) techniques, as e.g. in [8-11], based on the initial information theoretical analysis in [12]. Although such techniques can achieve significant capacity benefits, the DPC methods developed so far are complex as they require sophisticated signal processing at the transmitter. The fact that sphere-search algorithms [13] as complex as the ones used in MIMO detection [2,3] are employed for determining the data perturbation quantity, renders DPC techniques rather impractical in their present state. A promising alternative is the joint transmit-receive beamforming scheme as presented in [14] amongst others in the literature. Despite being less complex than DPC, the most robust beamforming schemes require iterative communication between the transmitter and receiver for the optimization of the joint processing and the system configuration. This needs to be done every time the channel characteristics change and hence, in fast fading environments, introduces considerable latency to the MIMO downlink system. Consequently, due to its advantageous tradeoff between performance and complexity, channel inversion precoding is considered a practical technique for contemporary MIMO downlink communications.

In this paper, following the theoretical results of Costa in [12], it is proposed that instead of using the knowledge of interference in MIMO systems to avoid it, a favourable approach would be to take advantage of the constructive part of this interference towards enhancing the useful signal at the receiver. In this direction, we introduce a simple and practical technique. Specifically, this paper proposes an improvement on the CI and RCI techniques presented in [6], [7] for systems employing PSK modulation. The proposed technique will be shown to be most effective at the lower signal to noise ratio (SNR) regions. The idea is based on the concept that for PSK, the instantaneous interference can be separated into constructive and destructive as previously presented in [15]. Although the analysis in [15] is done for multiple access interference (MAI) present in code division multiple access (CDMA) schemes, it can be modified and applied to the ICI that exists in MIMO systems as will be shown in this paper. By judiciously selecting the channels to be decorrelated and 
applying selective channel inversion, an amount of ICI which contributes beneficially to the instantaneous desired symbols is preserved at the MU received signal. Hence, the resulting instantaneous received SINR is enhanced without increasing the transmitted power per symbol. The fact that this instantaneous enhancement of SINR is performed at each symbol period results in an average received SINR improvement. It should be noted that the basic principle of interference exploitation that will be shown in the analysis can be extended to other, more complex MIMO precoding techniques. This contribution, however, is focused on CI precoding and presents some reference results for simple and practical precoding to reveal the potential of this concept on MIMO communication systems. The use of more intricate schemes could be the topic of further work. In the following, theoretical analysis and simulation results are provided to illustrate the superiority of the proposed technique.

\section{System Model And Conventional Channel INVERSION PRECODING}

The analysis presented in this and the following sections is with respect to the one shown in [7]. Since no signal enhancement processing is required at the individual MUs, the system configuration is irrespective to whether the receive antennas cooperate or not. Hence, a generic system of $N$ transmit (Tx) and $M$ receive ( $\mathrm{Rx}$ ) antennas is assumed here. The antennas can belong to one user or be shared by $K$ users, without any impact on the processing introduced. A frequency flat fading channel is supposed. As commonly suggested in the literature, the received signals of all antennas for the $i-$ th symbol period can be combined in a $M \times 1$ vector

$$
\mathbf{r}^{(i)}=\mathbf{H} \cdot \mathbf{x}^{(i)}+\mathbf{w}^{(i)}
$$

Here $\mathbf{r}^{(i)}=\left[r_{1}^{(i)}, r_{2}^{(i)}, \ldots r_{M}^{(i)}\right]^{T}$ and $\mathbf{H}$ is the $M \times N$ matrix that contains the frequency flat channel coefficients with the $(m, n)$ th element $h_{m, n}$ being the zero-mean unit-variance complexGaussian channel tap between the $n$-th transmitter and the $m$-th receiver. Also, $\mathbf{x}^{(i)}=\left[x_{1}^{(i)}, x_{2}^{(i)}, \ldots x_{N}^{(i)}\right]^{T}$ is the $N \times 1$ vector with the symbols transmitted by the place $N$ Tx antennas and $\mathbf{w}^{(i)}=\left[w_{1}^{(i)}, w_{2}^{(i)}, \ldots w_{N}^{(i)}\right]^{T}$ is the $M \times 1$ vector of the additive whit Gaussian noise (AWGN) components at the $M \mathrm{Rx}$ antennas. In conventional CI precoding the transmitted symbol vector is given as

$$
\mathbf{x}^{(i)}=f \cdot \mathbf{H}^{*} \cdot\left(\mathbf{H} \cdot \mathbf{H}^{*}\right)^{-1} \cdot \mathbf{b}^{(i)}
$$

where $\mathbf{b}^{(i)}=\left[b_{1}^{(i)}, b_{2}^{(i)}, \ldots b_{N}^{(i)}\right]^{T}$ is the $M \times 1$ data vector for the $i$-th symbol period, $\mathbf{T}=\mathbf{H}^{*} \cdot\left(\mathbf{H} \cdot \mathbf{H}^{*}\right)^{-1}$ is the pseudoinverse of the channel matrix and $\mathbf{H}^{*}$ denotes the Hermitian transpose of $\mathbf{H}$. $f$ is the scaling factor that ensures that $\left\|\mathbf{x}^{(i)}\right\|^{2}=1$ and is given as

$$
f=\sqrt{1 /\left(\mathbf{b}^{(i) *} \cdot\left(\mathbf{H} \cdot \mathbf{H}^{*}\right)^{-1} \cdot \mathbf{b}^{(i)}\right)}
$$

which derives instantaneous normalization of the transmitted power. For the case of RCI precoding applicable to systems with $N=M$ an MMSE-like precoding matrix $\mathbf{T}=\mathbf{H}^{*} \cdot(\mathbf{H}$. $\left.\mathbf{H}^{*}+a \cdot \mathbf{I}\right)^{-1}$ is applied and the transmitted vector is expressed as

$$
\mathbf{x}^{(i)}=f_{R} \cdot \mathbf{H}^{*} \cdot\left(\mathbf{H} \cdot \mathbf{H}^{*}+a \cdot \mathbf{I}\right)^{-1} \cdot \mathbf{b}^{(i)}
$$

where $\mathbf{I}$ is the identity matrix. The respective scaling factor as shown in [7] is given as

$$
f_{R}=\sqrt{1 / \operatorname{tr}\left[\frac{\boldsymbol{\Lambda}}{\mathbf{\Lambda}+a \cdot \mathbf{I}} \cdot \mathbf{Q}^{*} \cdot \mathbf{b}^{(i)} \cdot \mathbf{b}^{(i) *} \cdot \mathbf{Q}\right]}
$$

where $\operatorname{tr}[$.$] denotes the sum of the diagonal elements of a$ matrix, $\boldsymbol{\Lambda}$ is the eigenvalue matrix and $\mathbf{Q}$ is the unitary eigenvector matrix of the decomposition $\mathbf{H} \cdot \mathbf{H}^{*}=\mathbf{Q} \cdot \mathbf{\Lambda} \cdot \mathbf{Q}^{*}$. It can be seen that in this case the channel is not entirely orthogonalized and a certain amount of interference remains. By this configuration only the average power of the residue ICI can be controlled by the regularization factor $a$. It is shown in [7] that the value that maximizes the received SINR is $a=M \sigma^{2}$, where $\sigma^{2}$ is the noise variance at the receiver.

\section{ICI CHARACTERIZATION}

In this section we shall demonstrate the separation between constructive and destructive interference in MIMO systems. This is an expansion of the analysis presented in [15] for CDMA communications and therefore it is directly linked to it. This relation comes from the fact that channel taps in MIMO systems have an effect similar to the one from spreading signatures in CDMA [16]. For reasons of simplicity, in what follows CI is considered, but extension to RCI is trivial. The matrix $\mathbf{R}=\mathbf{H} \cdot \mathbf{H}^{*}$ in (2) is the channel crosscorrelation matrix and can be viewed as the maximum ratio combined (MRC) [17] interference matrix. Hence the precoding in (2) can also be viewed as a decorrelation-type precoding where the channel crosscorrelation matrix is inverted in order to zero the off-diagonal elements of $\mathbf{R}$ and attain interference free symbols at the receivers. However, in some cases, when the instantaneous data is such that the interference is constructive, not all of the off-diagonal elements of $\mathbf{R}$ need be zeroed, as the received symbols can benefit from them.

Let us first examine the interference amongst the subchannels in a MIMO system. The symbol-to-symbol ICI from $b_{k}^{(i)}$ to $b_{u}^{(i)}$ that results from the element $\rho_{k, u}=\sum_{n=1}^{N} h_{k, n} \cdot h_{n, u}^{*}$ of matrix $\mathbf{R}$ at the $i$-th symbol period is expressed as

$$
I C I_{k, u}^{(i)}=b_{k}^{(i)} \cdot \rho_{k, u}
$$

while the cumulative ICI on $b_{u}^{(i)}$ from all interfering symbols can be expressed as

$$
I C I_{u}^{(i)}=\sum_{k=1, k \neq u}^{M} b_{k}^{(i)} \cdot \rho_{k, u}
$$

Fig. 1 shows the constellations for binary (BPSK) and quadrature (QPSK) modulation as well as possible received signals. The shaded regions denote the constructive interference areas. In general constructive is the ICI that contributes to the energy of the symbol of interest. In more practical terms constructive is the ICI that yields received signals that have increased distance from the decision thresholds. The mathematical expression of this rule translates differently to different PSK constellations. 

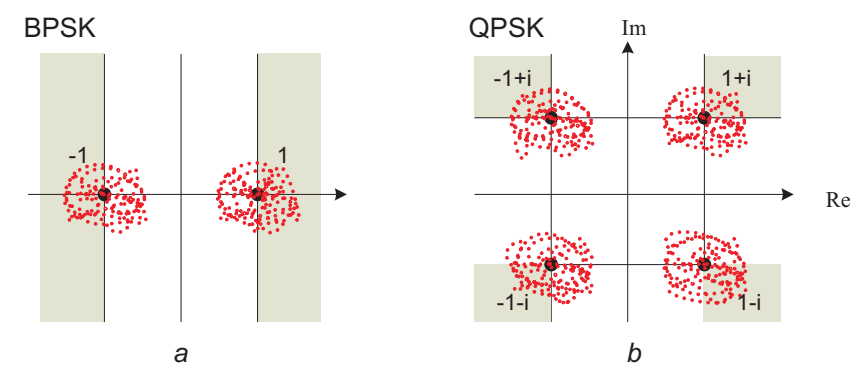

Fig. 1. The constructive interference regions in BPSK and QPSK constellations.

\section{A. ICI Characterization for BPSK modulation}

For BPSK modulation the desired symbol $b_{u}^{(i)} \in\{-1,+1\}$, so symbol-to-symbol ICI is constructive when it has the same sign as the desired data $b_{u}^{(i)}$ denoted by the shadowed part of the constellation in Fig. 1a.

$$
I C I_{k, u}^{(i)} \cdot b_{u}^{(i)}=b_{k}^{(i)} \cdot b_{u}^{(i)} \cdot \rho_{k, u}>0
$$

Similarly, the cumulative ICI is constructive when

$$
I C I_{u}^{(i)} \cdot b_{u}^{(i)}=b_{u}^{(i)} \cdot \sum_{k=1, k \neq u}^{M} b_{k}^{(i)} \cdot \rho_{k, u}>0
$$

Hence, in order to evaluate the interference between the symbols at each symbol period the instantaneous data need to be taken into account and the following matrix needs to be formed

$$
\begin{aligned}
\mathbf{G}^{(i)} & =\operatorname{diag}\left(\mathbf{b}^{(i)}\right) \cdot \mathbf{R} \cdot \operatorname{diag}\left(\mathbf{b}^{(i)}\right) \\
& =\left(\begin{array}{ccc}
\rho_{1,1} & b_{1}^{(i)} b_{2}^{(i)} \rho_{1,2} \ldots \cdots & b_{1}^{(i)} b_{M}^{(i)} \rho_{1, M} \\
b_{1}^{(i)} b_{2}^{(i)} \rho_{2,1} & \rho_{2,2} & b_{1}^{(i)} b_{M}^{(i)} \rho_{2, M} \\
\vdots & \ddots & \vdots \\
b_{M}^{(i)} b_{1}^{(i)} \rho_{M, 1} & b_{M}^{(i)} b_{2}^{(i)} \rho_{M, 2} \ldots & \rho_{M, M}
\end{array}\right)
\end{aligned}
$$

In (10) it is assumed that $\left\|b_{m}^{(i)}\right\|^{2}=1$. For regularized CI the same analysis can be applied by using $\mathbf{R}=\mathbf{H} \cdot \mathbf{H}^{*}+\alpha \cdot \mathbf{I}$ as presented in [7]. It is evident that each element of matrix $\mathbf{G}^{(i)}$ is in the form of the left part of (8) and hence denotes the symbol-to-symbol ICI. The sum of the off-diagonal elements of each row is in the form of the left part of (9) denoting the cumulative ICI. Therefore, by observation of $\mathbf{G}^{(i)}$ both cumulative and symbol-to-symbol ICI can be evaluated. The criteria of (8) and (9) translate respectively to

$$
\begin{gathered}
\mathbf{G}_{k, u}^{(i)}>0 \\
\operatorname{sum}\left(\left[\mathbf{G}^{(i)}-\operatorname{diag}\left(\mathbf{G}^{(i)}\right)\right]_{u}\right)>0
\end{gathered}
$$

where $[.]_{u}$ denotes the $u$-th row of a matrix. From the above it is apparent that according to the instantaneous data each element in (10) can derive constructive or destructive ICI. Since the former contributes to the signal power, the effective instantaneous received SINR instead of the conventional expression can be written as

$$
S I N R_{e}=\frac{S+\left\|I C I_{\text {constructive }}\right\|^{2}}{\left\|I C I_{\text {destructive }}\right\|^{2}+N_{n}}
$$

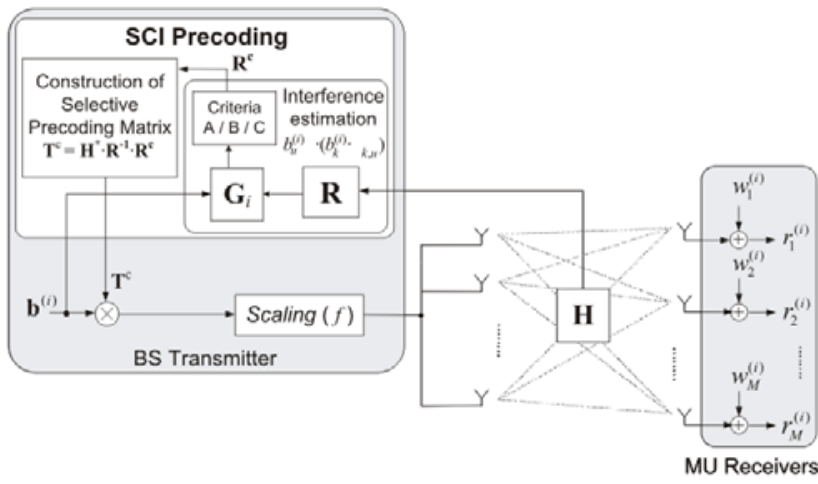

Fig. 2. The proposed SCI in the MIMO downlink.

where $S$ denotes the desired signal's energy and $N_{n}$ denotes the noise variance. Clearly, by allowing an amount of constructive and eliminating destructive interference, higher SINR values can be delivered to the MU receivers.

\section{B. ICI Characterization for QPSK modulation}

For QPSK modulation the desired symbol $b_{u}^{(i)} \in$ $\{( \pm 1 \pm i) / \sqrt{2}\}$. Therefore, the ICI needs to yield increased distances from the thresholds both on the real and imaginary axes in order to qualify as constructive, as depicted in the shadowed part of the QPSK constellation diagram in Fig. 1b. Hence, for the evaluation of ICI in this case the real and imaginary parts of ICI have to be considered separately, in order to characterize the interference. The constructive criteria of (11)-(12) can be applied to $\operatorname{Re}\left\{b_{u}^{(i)}\right\}$ and $\operatorname{Im}\left\{b_{u}^{(i)}\right\}$ separately, leading to the criteria

$$
\begin{gathered}
\mathbf{G}_{R E A L}^{(i)}>0 \quad \& \quad \mathbf{G}_{I M A G}^{(i)}>0 \\
\operatorname{sum}\left(\left[\mathbf{G}_{R E A L}^{(i)}-\operatorname{diag}\left(\mathbf{G}_{R E A L}^{(i)}\right)\right]_{u}\right)>0 \& \\
\operatorname{sum}\left(\left[\mathbf{G}_{I M A G}^{(i)}-\operatorname{diag}\left(\mathbf{G}_{I M A G}^{(i)}\right)\right]_{u}\right)>0
\end{gathered}
$$

where

$$
\begin{gathered}
\mathbf{G}_{R E A L}^{(i)}=\operatorname{Re}\left\{\operatorname{diag}\left(\mathbf{b}^{(i)}\right) \cdot \mathbf{R}\right\} \cdot \operatorname{Re}\left\{\operatorname{diag}\left(\mathbf{b}^{(i)}\right)\right\}, \\
\mathbf{G}_{I M A G}^{(i)}=\operatorname{Im}\left\{\operatorname{diag}\left(\mathbf{b}^{(i)}\right) \cdot \mathbf{R}\right\} \cdot \operatorname{Im}\left\{\operatorname{diag}\left(\mathbf{b}^{(i)}\right)\right\}
\end{gathered}
$$

\section{Selective Channel Inversion (SCI) Precoding}

\section{A. Modification of Precoding Matrix}

The above analysis can be exploited in practical CI precoding as shown in Fig. 2. Using channel state information (CSI) and knowledge of all symbols to be transmitted, readily available at the BS downlink, the interference to each received symbol can be estimated prior to transmission to provide matrix $\mathbf{G}^{(i)}$. By observation of the matrix $\mathbf{G}^{(i)}$ the elements of the crosscorrelation matrix $\mathbf{R}$ to be removed via decorrelation can be determined. Therefore, a constructive crosscorrelation matrix $\mathbf{R}^{c}$ can be formed that contains the elements of $\mathbf{R}$ that yield constructive interference according to the observation of $\mathbf{G}^{(i)}$ at every symbol period. Subsequently, instead of 
minimizing the MMSE as expressed for conventional CI and RCI

$$
J=E\left\{\left\|\mathbf{b}^{(i)}-\left(\mathbf{H} \cdot \mathbf{T} \cdot \mathbf{b}^{(i)}+\mathbf{w}^{(i)}\right)\right\|^{2}\right\}
$$

yielding $\mathbf{T}=\mathbf{H}^{*} \cdot \mathbf{R}^{-1}$, the following modified MMSE optimization could be applied

$$
J=E\left\{\left\|\mathbf{R}^{c} \cdot \mathbf{b}^{(i)}-\left(\mathbf{H} \cdot \mathbf{T}^{c} \cdot \mathbf{b}^{(i)}+\mathbf{w}^{(i)}\right)\right\|^{2}\right\}
$$

This MMSE expression leads to

$$
\mathbf{T}^{c}=\mathbf{H}^{*} \cdot \mathbf{R}^{-1} \cdot \mathbf{R}^{c} .
$$

which yields a transmitted signal as

$$
\mathbf{x}^{(i)}=f_{c} \cdot \mathbf{H}^{*} \cdot\left(\mathbf{H} \cdot \mathbf{H}^{*}\right)^{-1} \cdot \mathbf{R}^{c} \cdot \mathbf{b}^{(i)}
$$

It is obvious that $\mathbf{T}^{c}=\mathbf{T} \cdot \mathbf{R}^{c}$ consists of fixed block level (conventional) beamforming $\mathbf{T}$ combined with a symbol-bysymbol adaptive supplement $\mathbf{R}^{c}$. Moreover, as will be shown in the following section, apart from the formation of $\mathbf{R}^{c}$, the precoding operation is still linear, which makes it more easily applicable than DPC. Furthermore this technique does not involve iterative processing and communication between BS and MUs and therefore it can be evaluated as advantageous compared to joint Tx-Rx beamforming. It is evident from the above that the scaling factor needs to be modified for this new precoding matrix to

$$
f_{c}=\sqrt{1 / \operatorname{tr}\left[\mathbf{R}^{c *} \cdot\left(\mathbf{H} \cdot \mathbf{H}^{*}\right)^{-1} \cdot \mathbf{R}^{c}\right]}
$$

which ensures that $E\left(\left\|\mathbf{x}^{(i)}\right\|^{2}\right)=1$. Unlike [7] and in (3), (5), we chose to apply average normalization of the transmitted power by (21). The received symbols of the proposed precoding can be derived as

$$
\mathbf{r}^{(i)}=f_{c} \cdot \mathbf{R}^{c} \cdot \mathbf{b}^{(i)}+\mathbf{w}^{(i)}
$$

where the symbols of interest, due to $\mathbf{R}^{c}$, are corrupted by ICI that according to the observation of $\mathbf{G}^{(i)}$ is constructive. With conventional CI this constructive ICI that already exists in the system is wasted as all ICI is entirely removed. The received symbols in (22) have higher instantaneous SINR in (13) and provide more reliable detection than conventional CI.

\section{B. Criteria for Formation of Constructive Crosscorrelation Matrix}

The construction of matrix Rc is crucial to the performance enhancement. In [15] three criteria were presented for this procedure, which can be modified to apply on MIMO systems as will be briefly presented below. The criteria developed below are orientated to QPSK modulation on which most of the simulations are based.
1) SCI criterion A (SCI-A): The first criterion applies full orthogonalization on the symbols that experience destructive cumulative ICI and leaves the symbols that expect constructive ICI correlated. This could be expressed as:

$$
\begin{aligned}
& \text { Loop for } u=1 \text { to } M \\
& \text { If }\left(\begin{array}{c}
\operatorname{sum}\left(\left[\mathbf{G}_{R E A L}^{(i)}-\operatorname{diag}\left(\mathbf{G}_{R E A L}^{(i)}\right)\right]_{u}\right)<0 \text { or } \\
\operatorname{sum}\left(\left[\mathbf{G}_{I M A G}^{(i)}-\operatorname{diag}\left(\mathbf{G}_{I M A G}^{(i)}\right)\right]_{u}\right)<0
\end{array}\right) \\
& \text { then } \mathbf{R}_{k, u}^{c}=0 \text { for all } k \neq u \\
& \text { Else } \mathbf{R}_{k, u}^{c}=\rho_{k, u} \text { for all } k \neq u
\end{aligned}
$$

Here $\mathbf{R}_{k, u}^{c}$ is the $(k, u)$-th element of matrix $\mathbf{R}^{c}$.

2) SCI criterion $B(S C I-B)$ : An alternative to the above is to orthogonalize every symbol but only to the symbols that impose destructive interference to the useful signal. This means that the corresponding of all the negative elements in $\mathbf{G}^{(i)}$ are set to zero in $\mathbf{R}^{c}$. This could be expressed as:

$$
\begin{aligned}
& \text { Loop for } u=1 \text { to } M \\
& \text { Loop for } k=1 \text { to } M, k \neq u \\
& \text { If }\left(\left[\mathbf{G}_{R E A L}^{(i)}\right]_{k, u}<0 \text { or }\left[\mathbf{G}_{I M A G}^{(i)}\right]_{k, u}<0\right) \\
& \text { then } \mathbf{R}_{k, u}^{c}=0 \\
& \text { Else } \mathbf{R}_{k, u}^{c}=\rho_{k, u}
\end{aligned}
$$

With this criterion all destructive ICI is eliminated while pure constructive ICI is preserved.

3) SCI criterion $C(S C I-C)$ : The third criterion provides an optimization between residue ICI and processing complexity. This could be done by orthogonalizing only the symbols experiencing destructive cumulative ICI and only to the symbols that impose destructive ICI on them:

$$
\begin{aligned}
& \text { Loop for } u=1 \text { to } M \\
& \text { If }\left(\begin{array}{c}
\operatorname{sum}\left(\left[\mathbf{G}_{R E A L}^{(i)}-\operatorname{diag}\left(\mathbf{G}_{R E A L}^{(i)}\right)\right]_{y}\right)<0 \text { or } \\
\operatorname{sum}\left(\left[\mathbf{G}_{I M A G}^{(i)}-\operatorname{diag}\left(\mathbf{G}_{I M A G}^{(i)}\right)\right]_{u}\right)<0
\end{array}\right) \\
& \text { then } \\
& \text { loop for } k=1 \text { to } M, k \neq u: \\
& \quad \text { If }\left(\left[\mathbf{G}_{R E A L}^{(i)}\right]_{k, u}<0 \text { or }\left[\mathbf{G}_{I M A G}^{(i)}\right]_{k, u}<0\right) \\
& \text { then } \quad \mathbf{R}_{k, u}^{c}=0 \\
& \text { Else } \quad \mathbf{R}_{k, u}^{c}=\rho_{k, u} \\
& \text { Else } \mathbf{R}_{k, u}^{c}=\rho_{k, u} \text { for all } k \neq u
\end{aligned}
$$

It will be shown in the simulation sections that SCI$\mathrm{B}$ and SCI-C, provide quite similar performance with one outperforming the other in particular scenarios due to the differences in the residue ICI and in the resulting corresponding scaling factors. It should also be noted that the analysis for RCI is similar to the above and can be done by using $\mathbf{R}=\mathbf{H} \cdot \mathbf{H}^{*}+\alpha \cdot \mathbf{I}$ in the above calculations. However, due to the selective precoding a new interference environment is yielded for the received symbols and the regularization parameter $\alpha$ could be re-optimized. Nonetheless, in the selective RCI (SRCI) simulations that follow we have chosen to apply the analysis for $\mathbf{R}=\mathbf{H} \cdot \mathbf{H}^{*}+\alpha \cdot \mathbf{I}$ without reoptimizing $\alpha$. This might derive suboptimal performance but a major increase in complexity is circumvented by avoiding adapting parameter $\alpha$ for every system scenario. 
TABLE I

COMPLEXITY IN NUMBERS OF OPERATIONS PER FRAME FOR CI AND SCI.

\begin{tabular}{|c|c|c|c|}
\hline CI & No. of operations & SCI & No. of operations \\
\hline construct $R \times \mathbb{N}$ & $\mathbb{N} \cdot O\left(N \cdot M^{2}\right)$ & construct $\mathrm{R} \times \mathbb{N}$ & $\mathbb{N} \cdot O\left(N \cdot M^{2}\right)$ \\
\hline invert $R \times \mathbb{N}$ & $\mathbb{N} \cdot O\left(M^{2}\right)$ & invert $\mathrm{R} \times \mathbb{N}$ & $\mathbb{N} \cdot O\left(M^{3}\right)$ \\
\hline multiply $\mathrm{R}^{-1}$ with $b^{(i)} \times N_{f}$ & $N_{f} \cdot O\left(M^{2}\right)$ & construct $R^{c} \times N_{f}$ & $N_{f} \cdot O\left(M^{3}\right)$ \\
\hline multiply with $H^{*} \times N_{f}$ & $N_{f} \cdot O(N \cdot M)$ & multiply $R^{c}$ with $b^{(i)} \times N_{f}$ & $N_{f} \cdot O\left(M^{2}\right)$ \\
\hline calculate scaling factor $\times \mathbb{N}$ & $\mathbb{N} \cdot O(M)$ & multiply with $R^{-1} \times N_{f}$ & $N_{f} \cdot O\left(M^{2}\right)$ \\
\hline & & multiply with $H^{*} \times N_{f}$ & $N_{f} \cdot O(N \cdot M)$ \\
\hline & & calculate scaling factor $\times \mathbb{N}$ & $\mathbb{N} \cdot O(M)$ \\
\hline Total & $\mathbb{N} \cdot\left[O\left(M^{3}+O\left(N \cdot M^{2}\right)\right]+\right.$ & Total & $\mathbb{N} \cdot\left[O\left(M^{3}\right)+O\left(N \cdot M^{2}\right)\right]+$ \\
& $N_{f} \cdot\left[O\left(M^{2}+O(N \cdot M)\right]\right.$ & & $N_{f} \cdot\left[O\left(M^{3}\right)+\right.$ \\
& & & $\left.2 \cdot O\left(M^{2}\right)+O(N \cdot M)\right]$ \\
\hline
\end{tabular}

\section{Complexity Evaluation}

Complementary to the system analysis, a complexity evaluation is required to determine the practicality of the presented scheme. In order to investigate the complexity repercussions of the above methodology, a comparison of the conventional $\mathrm{CI}$ and proposed SCI technique is illustrated in Table 1. Here $N_{f}$ is the frame length and it is assumed that the channel estimates and consequently $\mathbf{R}$ need to be updated $\mathbb{N}$ times per data frame. For the slow fading case the updates need to be provided once per frame so $\mathbb{N}=1$, while for fast fading the updates are much more frequent and $1<<\mathbb{N} \leq N_{f}$. The complexity count is based on the total number of operations per frame. In the values for total number of operations the common analysis found in the literature (e.g. [18]) is followed where only the principal factors $O($.$) are included as they are$ the ones that essentially impact on the complexity. From the comparison it can be seen that the complexity impact on SCI is due to the need to reconstruct $\mathbf{R}^{c}$ on a symbol-by-symbol basis, which adds an overhead of $N_{f} \cdot\left[O\left(M^{3}\right)+O\left(M^{2}\right)\right]$ per frame. This imposes a relative complexity overhead

$$
F_{c}=\frac{N_{f} \cdot\left[O\left(M^{3}\right)+O\left(M^{2}\right)\right]}{\mathbb{N} \cdot\left[O\left(M^{3}\right)+O\left(N \cdot M^{2}\right)\right]+N_{f} \cdot\left[O\left(M^{2}\right)+O(N \cdot M)\right]}
$$

Some practical values of numbers of operations are shown in Table 2 for representative cases of $N=M=4, N=M=$ 10 and $N=M=16$. In all cases it is assumed that $N_{f}=100$ and $\mathbb{N}=1$ for slow fading, $\mathbb{N}=10$ for fast fading. It is evident that for $\mathbb{N}=1$ there is a non-trivial overhead, which drastically reduces when more frequent channel updates are required and $\mathbb{N}$ increases. This is because the inversion of $\mathbf{R}$ needs to be done more frequently for both $\mathrm{CI}$ and SCI and the construction of $\mathbf{R}^{c}$ for SCI imposes less relative complexity overhead.

It should be noted, though, that the complexity increase discussed involves the BS where resources are more affordable and complexity is less of a hindrance. Overall, the trade-off between performance and complexity is favourable for SCI as suggested by the simulation results that follow.

\section{UPPER Bound OF The SINR PER ReceIVED SYMBOL}

In this section a calculation of an upper bound for the SINR per received symbol of SCI for QPSK is attempted in order to provide a qualitative measure of the expected experimental performance. For the presented analysis, inline with common
TABLE II

PRACTICAL VALUES OF NUMBERS OF OPERATIONS FOR CONVENTIONAL AND SELECTIVE PRECODING FOR $N_{f}=100, \mathbb{N}=1$ SLOW FADING, $\mathbb{N}=10$ FAST FADING.

\begin{tabular}{|l|l|l|}
\hline & $\boldsymbol{C I}$ & $\boldsymbol{S} \boldsymbol{C} \boldsymbol{I}$ \\
\hline$N=M=4$, slow fading & $O\left(3.3 \times 10^{3}\right)$ & $O\left(1.1 \times 10^{4}\right)$ \\
\hline$N=M=4$, fast fading & $O\left(4.4 \times 10^{3}\right)$ & $O\left(1.2 \times 10^{4}\right)$ \\
\hline$N=M=10$, slow fading & $O\left(2 \times 10^{4}\right)$ & $O\left(1.3 \times 10^{5}\right)$ \\
\hline$N=M=10$, fast fading & $O\left(4 \times 10^{4}\right)$ & $O\left(1.5 \times 10^{5}\right)$ \\
\hline$N=M=16$, slow fading & $O\left(6 \times 10^{4}\right)$ & $O\left(5 \times 10^{5}\right)$ \\
\hline$N=M=16$, fast fading & $O\left(1.3 \times 10^{5}\right)$ & $O\left(5.7 \times 10^{5}\right)$ \\
\hline
\end{tabular}

practice, a normalized uncorrelated Rayleigh flat fading channel such that $\left\|\mathbf{H}_{u}\right\|^{2}=1$ is assumed where $\mathbf{H}_{u}$ denotes the $u$-th row of the channel matrix. For simplicity and to ease the notation, the channel matrix is assumed to be square, that is $N=M$. According to the separation between constructive and destructive interference the received signal of (22) for the $u$-th rx antenna can be written as

$$
r_{u}^{(i)}=f_{c} \cdot b_{u}^{(i)} \cdot \rho_{u, u}+f_{c} \cdot \sum_{n=1}^{A_{c}} b_{n}^{(i)} \cdot \rho_{u, n}+f_{c} \cdot \sum_{k=1}^{A_{d}} b_{k}^{(i)} \cdot \rho_{u, k}+w_{u}^{(i)}
$$

where $A_{c}, A_{d}$ are the number of interfering antennas that impose constructive and destructive interference to the desired symbol respectively, after selective precoding on the $i$-th symbol period. It is clear that in (24) the first term represents the desired signal and the second and third terms represent constructive and destructive ICI respectively. The coefficients $A_{c}, A_{d}$ in (24) are relevant to the form of precoding used and the number of interference elements in $\mathbf{R}$ that are removed.

In the case of conventional precoding $A_{c}=0, A_{d}=0$ since full orthogonalization is performed, which means that single user performance is achieved with a reduced SNR due to the scaling factor. For selective precoding the coefficients take variable values according to the method used. In the case of SCI-B where only constructive ICI is held $A_{d}=0$. This case serves as an upper bound of the SINR. For the other two methods these factors take values in the region $\{0, N-1\}$ such that $A_{c}>A_{d}$ so the SINR is enhanced compared to conventional precoding. For SCI-A, SCI-C it can be viewed by the analysis of section IV-B) that $A_{d}$ is the same for both criteria, but $A_{c}$ is larger for SCI-C than for SCI-A yielding better performance.

According to (24) and (13) and assuming statistically independent data the effective SINR per received symbol can be 
approximated by

$$
\begin{aligned}
\gamma & =\frac{\mathrm{E}\left\{D_{u}\right\}+\mathrm{E}\left\{I C I_{u}^{c}\right\}}{\mathrm{E}\left\{I C I_{u}^{d}\right\}+N_{0} / 2} \\
& =\frac{\mathrm{E}\left\{f_{c}^{2}\right\} \cdot\left[E_{s} \cdot \mathrm{E}\left\{\left|\rho_{u, u}\right|^{2}\right\}+\mathrm{E}\left\{A_{c}\right\} \cdot E_{s} \cdot \mathrm{E}\left\{\left|\rho_{u, k}\right|^{2}\right\}\right]}{\mathrm{E}\left\{f_{c}^{2}\right\} \cdot \mathrm{E}\left\{A_{d}\right\} \cdot E_{s} \cdot \mathrm{E}\left\{\left|\rho_{u, k}\right|^{2}\right\}+N_{0} / 2}
\end{aligned}
$$

where $I C I_{u}^{c}$ and $I C I_{u}^{d}$ denote the constructive and destructive part of ICI on the $u$-th antenna's received symbol respectively, $E_{s}$ is the transmitted energy per symbol and $\sigma^{2}$ $=N_{0} / 2$ is AWGN variance.

To evaluate $\gamma$ let us first determine $\mathrm{E}\left\{A_{c}\right\}, \mathrm{E}\left\{A_{d}\right\}$ of (25). By defining the probability of the ICI between a pair of antennas being constructive as $P_{c}$, for a generic MRC system with $N$-1 interfering antennas without selective precoding we have $\mathrm{E}\left\{A_{c}\right\}=P_{c} \cdot(N-1)$ and $\mathrm{E}\left\{A_{d}\right\}=\left(1-P_{c}\right) \cdot(N-1)$. For QPSK, as seen from (14)-(16) we have

$$
P_{c}=P\left(\begin{array}{l}
\operatorname{Re}\left(b_{u}\right) \cdot \operatorname{Re}\left(I C I_{u}\right)>0 \\
\operatorname{Im}\left(b_{u}\right) \cdot \operatorname{Im}\left(I C I_{u}\right)>0
\end{array}\right)
$$

For large numbers of antennas $N, M$ the interference can be approximated by a Gaussian random distribution. Real and imaginary parts of ICI can be assumed statistically independent and hence we have

$P_{c}=P\left(\operatorname{Re}\left(b_{u}\right) \cdot \operatorname{Re}\left(I C I_{u}\right)>0\right) \cdot P\left(\operatorname{Im}\left(b_{u}\right) \cdot \operatorname{Im}\left(I C I_{u}\right)>0\right)$

$$
\begin{aligned}
& \text { and } \\
& \begin{array}{r}
P\left(\operatorname{Re}\left(b_{u}\right) \cdot \operatorname{Re}\left(I C I_{u}\right)>0\right)=P\left(\operatorname{Re}\left(b_{u}\right)>0, \operatorname{Re}\left(I C I_{u}\right)>0\right) \\
+P\left(\operatorname{Re}\left(b_{u}\right)<0, \operatorname{Re}\left(I C I_{u}\right)<0\right)
\end{array}
\end{aligned}
$$

Again for large $N, M$ the first component of the right part of (28) can be written as

$$
\begin{aligned}
P\left(\operatorname{Re}\left(b_{u}\right)>0, \operatorname{Re}\left(I C I_{u}\right)>0=\right. \\
P\left(\operatorname{Re}\left(b_{u}\right)>0\right) \cdot P\left(\operatorname{Re}\left(I C I_{u}\right)>0\right)
\end{aligned}
$$

For i.i.d. QPSK symbols $b_{u}$ we have $P\left(\lim \operatorname{Re}\left(b_{u}\right)>0\right)=1 / 2$ and for the ICI we have

$$
\begin{aligned}
P\left(\operatorname{Re}\left(I C I_{u}\right)>0\right)= & P\left(\operatorname{Re}\left(\rho_{k, u}\right) \cdot \operatorname{Re}\left(b_{k}\right)>0\right) \\
= & P\left(\operatorname{Re}\left(\rho_{k, u}\right)>0, \operatorname{Re}\left(b_{k}\right)>0\right) \\
& +P\left(\operatorname{Re}\left(\rho_{k, u}\right)<0, \operatorname{Re}\left(b_{k}\right)<0\right)
\end{aligned}
$$

Since the data is independent of the channel and for an uncorrelated Rayleigh fading channel, the real and imaginary pars of the channel tap coefficients and hence the crosscorrelation values can be positive or negative with equal probability. Thus we have

$$
\begin{aligned}
& P\left(\operatorname{Re}\left(\rho_{k, u}\right)>0, \operatorname{Re}\left(b_{k}\right)>0\right) \\
& \quad=P\left(\operatorname{Re}\left(\rho_{k, u}\right)>0\right) \cdot P\left(\operatorname{Re}\left(b_{k}\right)>0\right) \quad=\frac{1}{2} \cdot \frac{1}{2}=\frac{1}{4}
\end{aligned}
$$

By substituting back in the above equations and using the symmetry in the components of $P_{c}$ shown in (27) and (30) we find $P\left(\operatorname{Re}\left(b_{u}\right) \cdot \operatorname{Re}\left(I C I_{u}\right)>0\right)=1 / 2$ and $P_{c}=1 / 4$ which derives $E\left\{A_{c}\right\}=(N-1) / 4$ and $E\left\{A_{d}\right\}=3 \cdot(N-1) / 4$.

In order to calculate $\mathrm{E}\left\{\left|\rho_{u, k}\right|^{2}\right\}$ in (25) we refer to the properties of the normalized uncorrelated Rayleigh channel. The variance of each element of the channel matrix is given as

$$
\operatorname{var}\left(h_{u, k}\right)=1 / N
$$

which gives for the $k$-th row $\operatorname{var}\left(\mathbf{H}_{k}\right)=1$. The mean of $\rho_{u, k}$ is zero for the channel under investigation and therefore $\mathrm{E}\left\{\left|\rho_{u, k}\right|^{2}\right\}=\operatorname{var}\left(\rho_{u, k}\right)=\operatorname{var}\left(\sum_{n=1}^{N} h_{u, m} \cdot h_{k, m}^{*}\right)=$ $\sum_{n=1}^{N} \operatorname{var}\left(h_{u, m} \cdot h_{k, m}^{*}\right)$. For each element in the sum we have $\operatorname{var}\left(h_{u, m} \cdot h_{k, m}^{*}\right)=\operatorname{var}\left(h_{u, m}\right) \cdot \operatorname{var}\left(h_{k, m}\right)=1 / N^{2}$ since uncorrelated fading is assumed. Therefore for $\rho_{u, k}, u \neq k$ we have

$$
\operatorname{var}\left(\rho_{u, k}\right)=\sum_{n=1}^{N} 1 / N^{2}=1 / N
$$

and $\operatorname{var}\left(\rho_{u, u}\right)=\operatorname{var}\left(\mathbf{H}_{u}\right)=1$. The upper bound for the SINR per transmitted symbol is met when all destructive interference is removed $\left(A_{d}=0\right)$ while all constructive ICI is maintained. The SINR upper bound can hence be expressed using the above as

$$
\gamma \leq \frac{\mathrm{E}\left\{f_{c}^{2}\right\} \cdot E_{s} \cdot\left(1+\frac{N-1}{4 \cdot N}\right)}{\sigma^{2}}
$$

At this point it should be noted that the expectation of the scaling factor in (34) cannot be calculated analytically due to the fluctuations in the elements that are removed form matrix $\mathbf{R}$ on a symbol-by-symbol basis. However, simulations have indicated that the scaling factors offered by the proposed technique are related proportionally to the ones from conventional CI by $\mathrm{E}\left\{f_{c}^{2}\right\}=\frac{c}{N} \cdot \mathrm{E}\left\{f^{2}\right\}$, where $c$ is a constant dependent on the order of the PSK modulation used. The scaling factor of conventional CI has been well investigated in [19] where the scaling factor notation used is the inverse of the square of the scaling factor as given here $\beta=1 / f^{2}$ and it is shown that it follows a scaled $F$-distribution represented for $N=M$ by $\beta \sim N F_{2 N, 2}$ with a probability density function (pdf) of $p(\beta)=N \frac{(\beta)^{N-1}}{(1+\beta)^{N+1}}$. According to this we have

$$
\begin{aligned}
\mathrm{E}\left\{f^{2}\right\} & =\mathrm{E}\left\{\frac{1}{\beta}\right\} \\
& =N \int_{-\infty}^{\infty} \frac{1}{\beta} p(\beta) d \beta \\
& =N \int_{-\infty}^{\infty} \frac{\beta^{N-2}}{(1+\beta)^{N+1}} d \beta
\end{aligned}
$$


and (34) yields

$$
\gamma \leq \frac{c \cdot E_{s} \cdot\left(1+\frac{N-1}{4 \cdot N}\right) \cdot \int_{-\infty}^{\infty} \frac{\beta^{N-2}}{(1+\beta)^{N+1}} d \beta}{\sigma^{2}}
$$

The integral in (36) is given as

$$
\int_{-\infty}^{\infty} \frac{\beta^{N-2}}{(1+\beta)^{N+1}} d \beta=N \frac{\beta_{2}^{N-1} F_{1}(N-1, N+1, N,-\beta)}{N-1}
$$

where ${ }_{2} F_{1}(x, y, z, q)$ is the hypergeometric function, details of which can be found in [20]. By substituting in (36) analytical values of the SINR upper bound can be found according to this approach. A comparison of the analytical expression (36) of the upper bound of the received SINR to simulation results is given in Fig. 6 in the following.

\section{Numerical And Simulation Results}

Monte Carlo simulations of SCI and conventional CI and RCI, have been carried out for various numbers of antennas on frequency flat fading MIMO channels in order to present the relevant comparison. Both BPSK and QPSK modulation have been employed and unless stated otherwise perfect channel estimates are assumed. As in [7], in the results shown the SNR parameter $\rho=1 / \sigma^{2}$ is used, where $\sigma^{2}$ is the AWGN variance, in order to ease comparison. It is assumed that $N=M$ for the majority of the results shown. For the simulations for selective regularized CI (SRCI) matrix $\mathbf{R}=\mathbf{H} \cdot \mathbf{H}^{*}+\alpha \cdot \mathbf{I}$ was used in the analysis of section IV in order to derive the precoding matrix.

In Fig. 3 the average symbol error rate (SER) versus $\rho$ performance is shown for conventional and selective CI and RCI. A number $N=M=10$ antennas is assumed and QPSK modulation is employed. The results show an SNR gain of $4 \mathrm{~dB}$ for CI with SCI-B and SCI-C. For SRCI the benefit to RCI exists only in the lower SNR region up to $25 \mathrm{~dB}$. This is due to the fact that by regularizing the inverse of $\mathbf{R}$ an amount of interference is introduced which can not be manipulated for SRCI. This is more pronounced at higher SNRs where ICI becomes more prominent.

As the number of antennas increases the performance gain also rises. This is due to the fact that the increased space diversity introduces more interference elements which impede the performance of conventional CI but allow for better interference optimization with SCI. This is shown in Fig. 4 where the same comparison as in Fig. 3 is depicted for $N=M=16$. An SNR gain of $5 \mathrm{~dB}$ is achieved for SCI-B and SCI-C compared to CI, while the SNR region where SRCI is superior to RCI is increased.

The scenarios of non-square channel matrices for the cases of $N=15, M=10$ and $N=10, M=8$ are investigated in Fig. 5 for QPSK modulation. It should be noted that RCI does not apply to these cases and therefore results for RCI and SRCI are omitted. It can be seen that conventional CI shows a superior SER performance compared to the square channel case but still SCI provides performance benefits for these scenarios. A gain of $2 \mathrm{~dB}$ in the transmitted SNR can be viewed for both cases between CI and SCI-B and the $\mathrm{SER}=10^{-2}$ region.

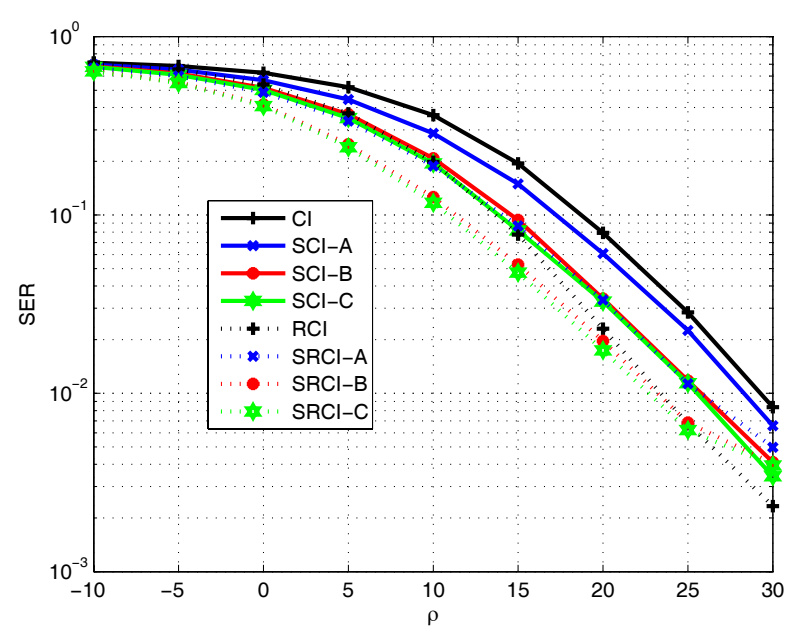

Fig. 3. SER performance for CI, RCI, SCI and SRCI for $N=M=10$, QPSK.

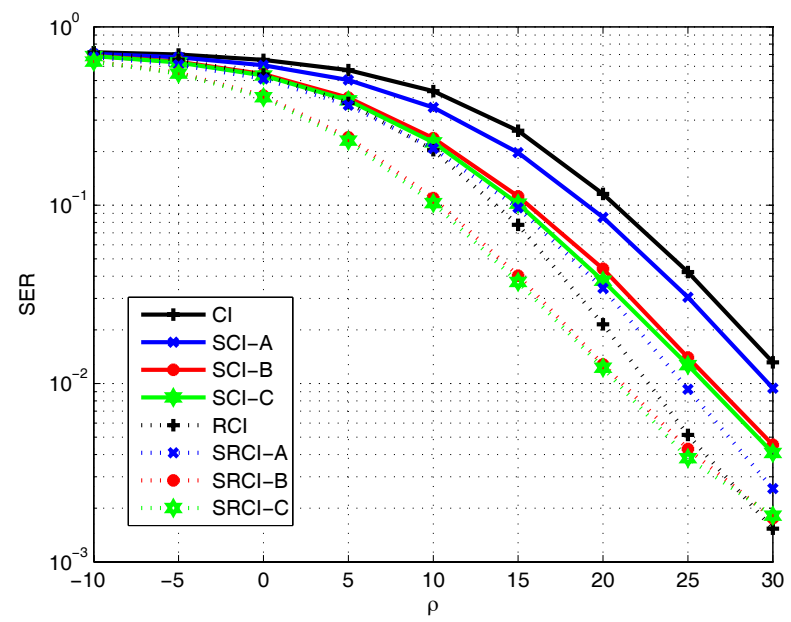

Fig. 4. performance for CI, RCI, SCI and SRCI for $N=M=16$, QPSK.

The analysis made in section III and VI for the received SINR is verified by simulation in Fig. 6 where the average received per symbol SINR is plotted against the average transmitted per symbol SNR for $N=M=10$ and QPSK. It can be observed that for CI and RCI the transmitted SNR values diminish to lower received SINR values as the interference introduced deteriorates the signal reliability. However for SCI the received SINR is higher than the transmitted SNR as the interference is controlled towards the enhancement of the useful symbols' energy. For SCI-A and SCI-C this applies only for the low SNR region as the curves saturate. This is because these methods allow an amount of destructive ICI which becomes dominant at high SNR where the noise is negligible. However, since SCI-B preserves only and purely the constrictive part of interference the corresponding curve shows no saturation and a significant SINR improvement is attained for all transmitted SNRs. The theoretical upper bound of the SINR which is achieved by employing SCI-B is also depicted in the figure and it can be seen that theoretical and simulation results match closely. 


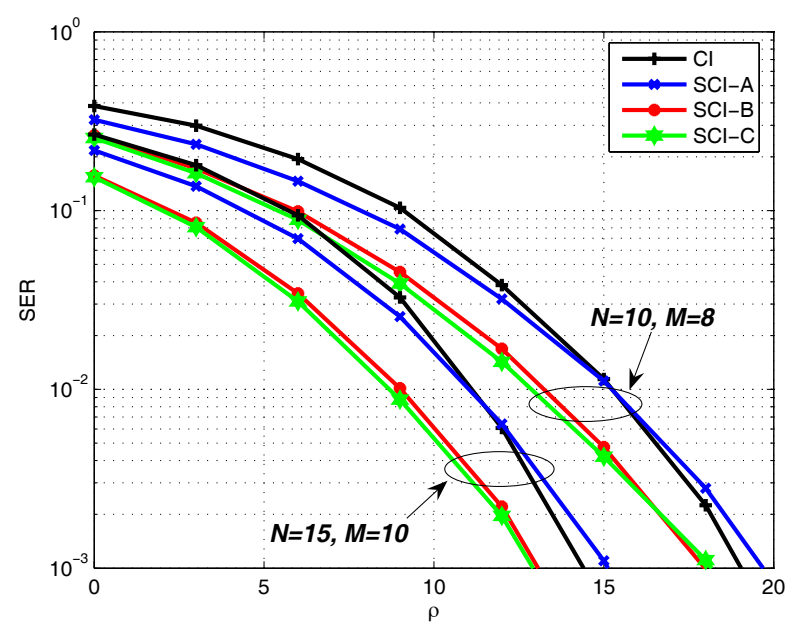

Fig. 5. SER performance for CI, SCI for the non-square channel matrix scenarios of $N=15, M=10$ and $N=10, M=8$, QPSK.

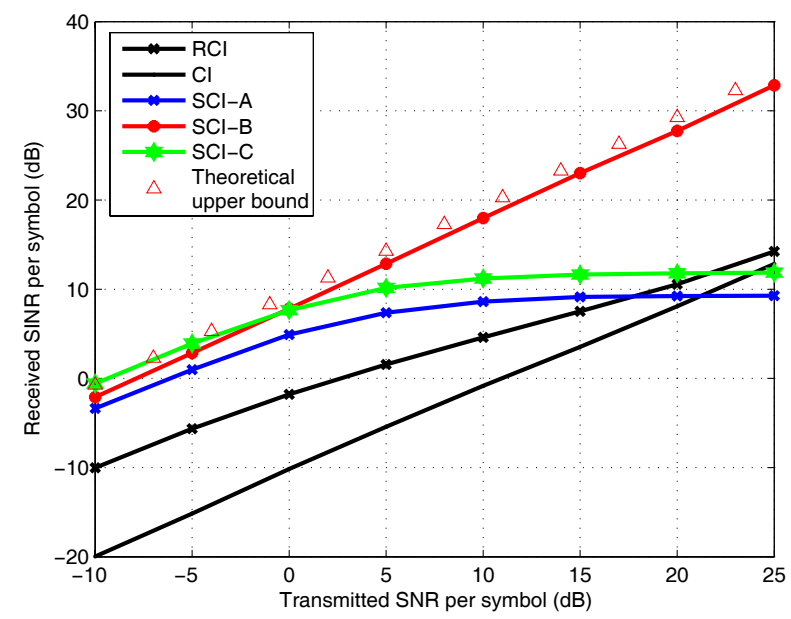

Fig. 6. Received SINR vs. transmitted SNR for CI, RCI, SCI for $N=M=$ 10, QPSK.

The throughput benefits of the proposed technique are examined in Fig. 7-8. In the results depicted, throughput is expressed as $T_{r}=(1-\mathrm{BLER}) \cdot m \cdot N$ bits/channel use, where BLER is the block error rate, $m=1 \mathrm{bit} / \mathrm{symbol}$ for BPSK and $m=2$ for QPSK. The block length used for these simulations is $N_{f}=100$ symbols. In Fig. 8 the case of $N=M=16$ is investigated and for QPSK, a constant transmit SNR gain of $4 \mathrm{~dB}$ in the values of $\rho$ can be observed for SCI and SRCI compared to CI and RCI respectively. This is magnified to $7 \mathrm{~dB}$ for BPSK, and it exists for up to $\rho=30 \mathrm{~dB}$ where all techniques attain almost optimum throughput. It is interesting to note that, for small values of $\rho$ up to $17 \mathrm{~dB}$, BPSK modulation provides better throughput than QPSK due to improved BLER and enhanced potential for interference exploitation, since BPSK constellation has larger margins for constructive ICI than QPSK, as seen if Fig. 1. This observation also justifies the selection of low order PSK for the investigated scenarios.

By increasing the number of antennas the throughput benefit is enhanced. This effect can be observed more clearly in

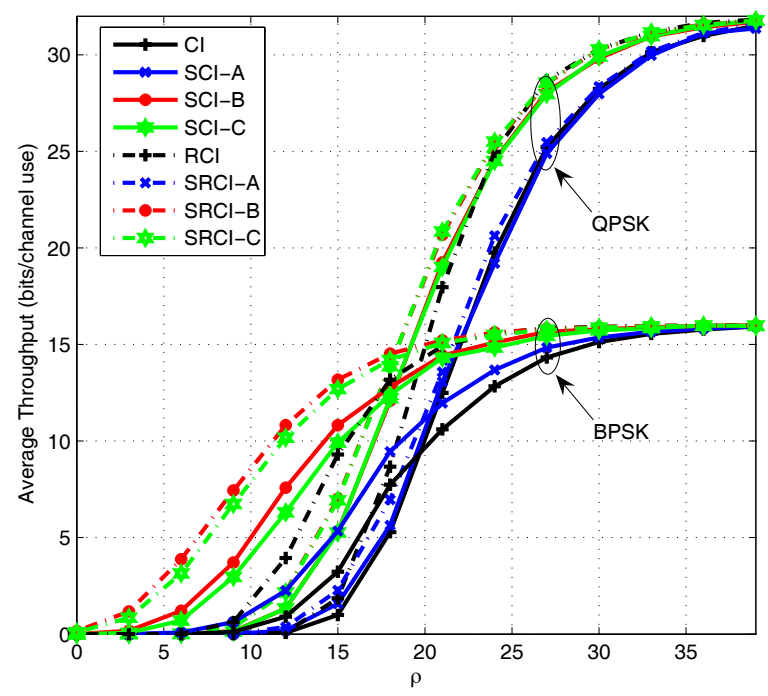

Fig. 7. Average Throughput vs. $\rho$ for CI, RCI, SCI and SRCI for $N=$ $M=16$.

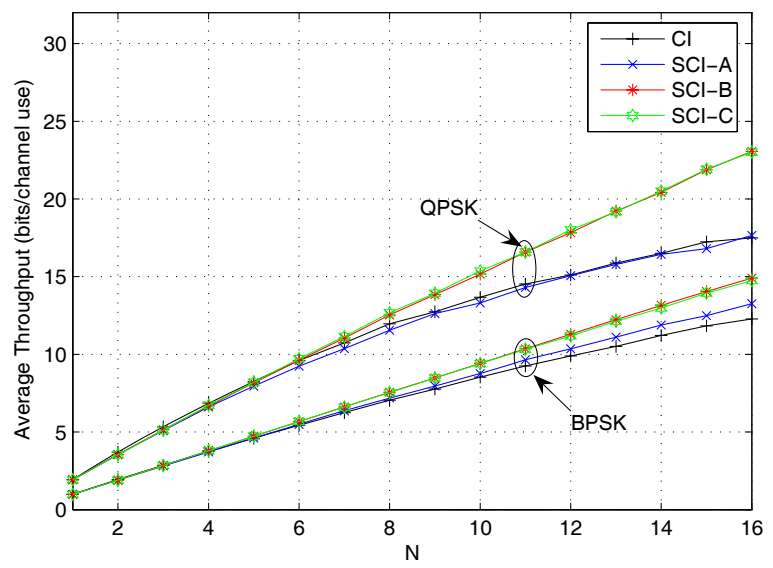

Fig. 8. Average Throughput vs. $N$ for CI, SCI for $\rho=23 \mathrm{~dB}$.

Fig. 8 where the throughput performance for increasing $N$ is depicted for $\rho=23 \mathrm{~dB}$. The gradient for the proposed schemes is greater and performance benefits are gained for all numbers of antennas. The difference in throughput grows with increased space diversity.

The methodology introduced above might suggest that the proposed technique has an increased sensitivity to channel estimation errors. Therefore, the performance of SCI was examined in systems where erroneous channel estimations are available. In Fig. 9 the scenario of Fig. $4(N=M=16$, QPSK) is examined with an error of $5 \%$ in the real and imaginary part of the channel coefficients. The SER results for perfect CSI are repeated for comparison. The CSI errors are generically modeled and no specific channel estimation method is assumed. A random Gaussian error is added to the real and imaginary part of the actual channel coefficients, with variance of $\mathrm{E}\left\{\left|\mathbf{H}_{\mathbf{u}}-\hat{\mathbf{H}}_{\mathbf{u}}\right| / \mathbf{H}_{\mathbf{u}}\right\}=5 \%$ of that of the channel taps, in order to attain the erroneous channel coefficients that are fed to the proposed precoding. The results show that 


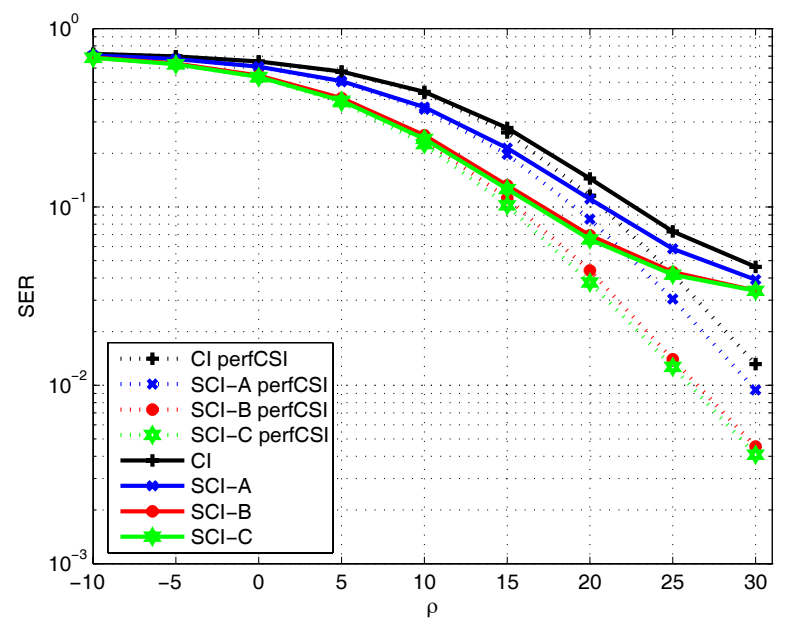

Fig. 9. SER performance for CI, SCI with 5\% CSI errors, QPSK.

the performance of conventional and proposed techniques is severely afflicted from this considerable error. However, the performance trend of the proposed scheme follows the one of the conventional, which suggests that SCI is as sensitive to channel estimation errors as $\mathrm{CI}$.

\section{CONCLUSIONS AND Future Work}

It was shown through theoretical analysis and simulations that by introducing selectivity in the orthogonalization between the channel elements in a downlink MIMO channel, SINR and performance benefits can be gleaned. The major contribution of this work is the proposal of an alternative translation of the information theoretical results in [12]. That is, exploiting part of the known interference inherent in a MIMO system towards the enhancement of the useful signal is an advantageous alternative to completely avoiding all interference. There exists a certain portion of interference that can contribute to the useful signal and when exploited on a symbol by symbol basis, it can enhance the reliability of the detection variables at the MU receivers.

\section{ACKNOWLEDGEMENTS}

This work has been jointly funded by EPSRC and Philips Research Labs, UK. The authors would like to thank Dr. Tim Moulsley for the helpful discussions throughout this research contribution.

\section{REFERENCES}

[1] G. Caire and S. Shamai, "On the achievable throughput of a multiantenna Gaussian broadcast channel," IEEE Trans. Inform. Theory, vol. 43, pp. 1691-1706, July 2003.

[2] E. Viterbo and J. Buotros, "A universal lattice code decoder for fading channels," IEEE Trans. Inform. Theory, vol. 45, pp.1639-1642, July 1999.

[3] O. Damen, A. Chkeif, and J. C. Belfiore, "Lattice code decoder for space-time codes," IEEE Commun. Lett., vol. 4, pp. 161-163, May 2000.

[4] G. J. Foschini, "Layered space-time architecture for wireless communication in a fading environment when using multi-element antennas," Bell Labs Tech. J., vol. 1, pp. 41-59, 1996.

[5] P. W. Wolniansky, G. J. Foschini, G. D. Golden, and R. A. Valenzuela, "V-BLAST: an architecture for realizing very high data rates over the rich-scattering wireless channel," in Proc. URSI ISSSE, 1998, pp. 295300 .
[6] T. Haustein, C. von Helmolt, E. Jorswieck, V. Jungnickel, and V. Pohl, "Performance of MIMO systems with channel inversion," in Proc. 55th IEEE Veh. Technol. Conf. (VTC), vol. 1, Birmingham, AL, May 2002, pp. 35-39.

[7] C. B. Peel, B. M. Hochwald, A. L. Swindlehurst, "A vector-perturbation technique for near-capacity multiantenna multiuser communication-part I: channel inversion and regularization," IEEE Trans. Commun., vol. 53, no. 1, pp. 195-202, Jan. 2005

[8] M. Tomlinson, "New automatic equalizer employing modulo arithmetic," Electron. Lett., vol. 7, no. 5,6, pp. 138-139, Mar. 1971.

[9] H. Harashima and H. Miyakawa, "Matched transmission technique for channels with intersymbol interference," IEEE Trans. Commun., vol. COM-20, pp. 774-780, Aug. 1972.

[10] U. Erez, S. Shamai, and R. Zamir, "Capacity and lattice strategies for cancelling known interference," IEEE Trans. Inform. Theory, vol. 51, no. 11, pp. 3820-3833, Nov. 2005.

[11] H. El Gamal, G. Caire, and M. Damen, "Lattice coding and decoding achieve the optimal diversity-multiplexing tradeoff of MIMO channels," IEEE Trans. Inform. Theory, vol. 50, pp. 968-985, June 2004.

[12] M. Costa, "Writing on dirty paper," IEEE Trans. Inform. Theory, vol. IT-29, pp. 439-441, May 1983.

[13] B. Hassibi and H. Vikalo, "On the expected complexity of integer least squares problems," in Proc. IEEE Int. Conf. Acoust., Speech, Signal Process., Orlando, FL, May 2002, pp. 1497-1500.

[14] R. Farrokhi, K. J. R. Liu, and L. Tassiulas, "Transmit beamforming and power control for cellular wireless systems," IEEE J. Select. Areas Commun., vol. 16, no. 8, pp. 1437-1450, Oct. 1998

[15] C. Masouros and E. Alsusa, "A novel transmitter-based selectiveprecoding technique for DS/CDMA systems," in Proc. IEEE Int. Conf. Commun. (ICC), June 2007.

[16] D. Gesbert, M. Shafi, D. Shiu, P. J. Smith, and A. Naguib, "From theory to practice: an overview of MIMO space-time coded wireless systems," IEEE J. Select. Areas Commun., vol. 21, no. 3, pp. 281-301, Apr. 2003

[17] T. K. Y. Lo, "Maximum ratio transmission," IEEE Trans. Commun., vol. 47, no. 10 , pp. $1458-1461$, Oct. 1999.

[18] M. G. Alkhansari and A. B. Gershman, "Fast antenna subset selection in MIMO systems," IEEE Trans. Signal Processing, vol. 52, no. 2, pp. 339-347, Feb. 2004

[19] X. Shao, J. Yuan, and Y. Shao, "Error performance analysis of linear zero forcing and MMSE precoders for MIMO broadcast channels," IET Commun., vol. 1, no. 5, Oct. 2007

[20] M. Abramowitz and I. A. Stegun, Handbook of Mathematical Functions: with Formulas, Graphics and Mathematical Tables. Wiley and Sons, New York, 1972.

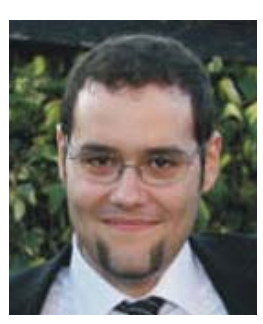

Christos Masouros (M'06), received his Diploma in Electrical \& Computer Engineering from the University of Patras, Patras, Greece, in 2004 and MSc by research in Electrical \& Electronic Engineering from the University of Manchester, Manchester, UK in 2006. He is currently pursuing his $\mathrm{PhD}$ degree in the Microwave and Communication Systems group of the University of Manchester. His research interests lie in the field of wireless communications and signal processing with particular focus on interference MIMO communications mitigation techniques for CDMA, multicarrier and

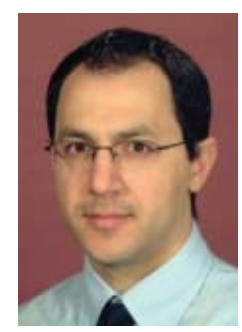

Emad Alsusa (M'06-SM'07), received BSc in Electrical \& Electronic Engineering from Salford University, Salford, UK, in 1996 and $\mathrm{PhD}$ in Electrical \& Electronic Engineering from Bath University, Bath, UK, in 2000. From June 2000 to September 2003 he was with the School of Engineering and Electronics at Edinburgh University as a Postdoctoral Research fellow. He joined Manchester University in 2003 as a Lecturer of Communication Engineering. He is a senior member of the IEEE. His research interests are in the area of wireless communication networks, especially, modulation and multiple access techniques, channel estimation, channel coding, interference mitigation, multiuser detection and MIMO techniques. 